\title{
Saving art in situ
}

\section{A conservation scientist explains how borrowing gadgets from Mars rovers helps preserve culture on Earth.}

Art conservation protects our cultural heritage by preventing loss. During the past 50 years, conservators have respected the material integrity of objects, adopting an approach of minimum intervention. Removing even small samples from works of art for scientific analysis is acceptable only when no other technique can provide the necessary information. Until recently, few analytical choices were available that did not necessitate sampling, but the development of non-invasive, portable instruments has greatly changed the art conservation landscape.

Conservation scientists apply analytical procedures to important works of art and architecture to establish what an object is made of and how it was made (technical art history), as well as when and where it was created (dating and provenance). Research also reveals how materials change over time, and suggests ways to slow their deterioration. This information helps art historians, archaeologists and conservators to better understand the objects they are studying and preserving.

Much is to be gained from bringing the analytical instrument from the laboratory to the work of art, rather than the reverse. It is inherently risky to move art objects: insurance companies charge high fees to cover damage during transportation, often prohibiting the movement of an object for study, and some items are simply not transportable. Portable instruments permit on-the-spot brainstorming among conservators, museum curators, art historians, site managers and others, allowing good solutions to be reached and such partnerships to be strengthened.

Many items of analytical equipment are now produced in miniaturized portable versions. One example is an X-ray fluorescence spectrometer, used to determine the elemental composition of a material. X-ray fluorescence has limitations because it cannot tell you the absolute quantities of each element, does not distinguish between various layers beneath the surface or show how the elements are combined to form molecules and crystals. But in its non-invasive and portable form, the technique is revolutionizing the conservation field.
Conservation science is too small a market to attract the analytical-instrument industry. Conservation scientists often rely on tools that have been developed for other fields, such as the medical, oil and automobile industries or homeland security, adapting the technologies to the needs of the art world. Recently, they have even looked to the instruments of space programmes and planetary exploration.

by an energy-dispersive detector that allows the diffraction and fluorescence signatures to be measured simultaneously in minutes. The combined diffraction and fluorescence data can differentiate not only between some common salts but also between hydration states, which may pinpoint the causes of deterioration and lead to the design of a suitable conservation strategy for an art object.

The new instrument will be extensively used to analyse the collection of antiquities, outdoor sculptures and manuscripts at the Getty Museum in Los Angeles. Later this year in the Roman ruins of Herculaneum, Italy, it will help examine excavated murals for pigments, salts and deterioration products. Other non-invasive portable instruments (such as Raman spectrometers and multi-spectral analysers) have been used to identify pigments and reveal inscriptions on the shroud of the Fayum mummy Herakleides, held at the Getty Museum. The additional shroud inscription 'son of Thermoutarion' was revealed only when light of a specific wavelength was used.

Of course, all portable instruments come with limitations. Miniaturization and portability may compromise accuracy and sensi-

For example, NASA has developed an innovative $\mathrm{X}$-ray diffraction and fluorescence instrument that will be deployed on Mars in 2010 as part of the Mars Science Laboratory rover. This technology was redeveloped for applications on Earth by the Californiabased company inXitu, and the firm's chief technology officer Philippe Sarrazin has collaborated with the Getty Conservation Institute, a programme of the J. Paul Getty Trust in Los Angeles, to build an adapted portable instrument for art analysis. The instrument was redesigned to reflect X-rays off a surface - thus avoiding the need for sample collection - rather than using transmission mode, which would require the $\mathrm{X}$-ray detector to be placed beneath the object surface.

On location, the instrument is adjusted in front of the artwork using precise laser alignment guides and a high-resolution camera. A fine X-ray beam is directed at the surface and the radiation scattered back is collected tivity. The user-friendly nature of portable instruments may convince non-experts to try to operate them, perhaps leading to incorrect analyses. Certain studies (such as stratigraphic analysis) necessitate cross-sections and therefore still require samples to be taken. Nevertheless, the use of such instruments will solve many key problems, such as analysing precious manuscripts without taking samples, or heavy statues, marble works and outdoor murals that cannot be moved.

To implement this technology skilfully, I foresee an increasing need for a new role in art conservation - the 'scientist-conservator'. Combining knowledge of artists' methods, the science of conservation and the technical instruments, these hybrid professionals will be able to ask the right questions to conserve our future.

Giacomo Chiari is chief scientist at the Getty Conservation Institute, 1200 Getty Center Drive, Los Angeles, California 90049-1684, USA. 